\title{
CARTOGRAFÍA DE LAS PÉRDIDAS POTENCIALES DE N-UREA POR VOLATILIZACIÓN EN SUELOS DE CHILE CENTRAL
}

\author{
Mapping potential losses of $\mathrm{N}$-urea by volatilization in soils of central Chile \\ Manuel Casanova P. ${ }^{1}$, Carlos Benavides Z. ${ }^{2}$ \\ ${ }^{1}$ Departamento de Ingeniería y Suelos. Facultad de Ciencias Agronómicas. Universidad \\ de Chile. Casilla 1004, Santiago. Correspondencia: mcasanov@uchile.cl. \\ ${ }^{2}$ Centro de Estudios de la Vid (CEVID). Universidad de Chile
}

\begin{abstract}
Available soil information from a soil cartography and a soil data base were used to predict the spatial distribution of ammonia volatilization by surface applied granular urea within the central zone of Chile, assuming that the potential losses depends only on soil properties, which are associated with the physical space known as microsite. Relevant properties (cation exchange capacity, urease activity, $\mathrm{pH}$ buffering capacity and soil $\mathrm{pH}$ post urea hydrolysis or $\mathrm{pH}_{f}$ ) on ammonia loss process were defined according to a conceptual model, determining threshold values for high potential ammonia losses: CEC $\leq 20 \mathrm{cmol}_{(+)}$ $\mathrm{kg}^{-1}$, urease activity $\geq 40 \mu \mathrm{g} \mathrm{N}$-urea $\mathrm{g}^{-1} \mathrm{~h}^{-1}$ and $\mathrm{pH}_{f} \geq 8.0$. On other hand, considering the use of some simplified pedotransfer functions, these relevant properties were predicted for the cartographic units of 82 soil Series, estimating around of 88,000 ha the area with the highest risk ammonia losses, corresponding principally to Mollisols.
\end{abstract}

Key Words: pedotransfer functions, urea, volatilization

\section{RESUMEN}

Empleando información cartografíca y una base de datos se predijo la distribución espacial de la volatilización a partir de urea granular aplicada superficialmente en la zona central de Chile, bajo el supuesto que el potencial de pérdida amoniacal sólo depende de las propiedades del suelo, y que se asocian al espacio físico conocido como micrositio. Las propiedades relevantes (capacidad de intercambio catiónico, actividad ureásica,

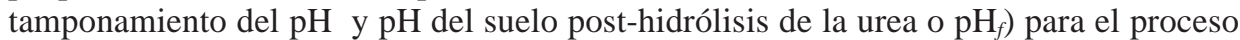
de pérdida de amoníaco, se definieron acorde a un modelo conceptual, determinándose que los valores umbrales para un alto potencial de pérdidas son: CIC $\leq 20 \mathrm{cmol}(+) \mathrm{kg}^{-1}$, actividad ureásica $\geq 40 \mu \mathrm{g} \mathrm{N}$-urea $\mathrm{g}^{-1} \mathrm{~h}^{-1}$ and $\mathrm{pH}_{f} \geq 8,0$. Por otra parte, teniendo en cuenta el uso de algunas funciones de pedotranferencia simplificadas, dichas propiedades relevantes fueron predichas para las unidades cartográficas de las 82 Series del suelo, estimándose en alrededor de 88.000 hectáreas el área con mayor riesgo de pérdidas de amoníaco, correspondiendo principalmente a Mollisoles.

Palabras Claves: funciones de pedotransferencia, urea, volatilización 


\section{INTRODUCCION}

Si bien la sustentabilidad es una meta de amplia aceptación, el paradigma dominante en la agricultura es que perdura un conflicto entre la obtención de altos rendimientos y la reducción de los impactos ambientales (Keller and Brummer, 2002). La intensificación de la agricultura ha aumentado notablemente la capacidad productiva de los agro-ecosistemas, pero ha tenido consecuencias indeseables para el ambiente, incluyendo la degradación de los suelos, de los recursos hídricos y, una alteración de los ciclos biogeoquímicos (Drinkwater and Snapp, 2007).

Se reconoce a la agricultura como la fuente principal de amoníaco atmosférico (Ji-Qin $\mathrm{Ni}$ and Heber, 2008), contribuyendo con un $50 \%$ de las emisiones globales (Schlesinger and Hartley, 1992). Las pérdidas de nitrógeno (N) debido a estas emisiones pueden significar a los agricultores una eficiencia reducida del fertilizante nitrogenado, contribuyendo a una más baja eficiencia total del nitrógeno aplicado; esto es, 50\% en los trópicos y $70 \%$ en zonas templadas (Malhi et al., 2001). Para evitar las consecuencias negativas de las pérdidas amoniacales, el agricultor tiende a aplicar fertilizante en exceso, incrementando las pérdidas, sus costos de producción y, al ser un gas químicamente activo que se deposita, causa acidificación y eutroficación de los ecosistemas naturales (Fenn et al., 2003; Galloway et al., 2003).

Globalmente, la urea es el fertilizante nitrogenado sólido más barato y más empleado para la producción agrícola. Su utilización en aplicaciones en cobertera constituye una forma de manejo normalmente no recomendada, al existir condiciones particulares en las cuales esta fuente presenta una efectividad inherente baja; aconsejándose su incorporación para evitar las pérdidas amoniacales (Fenn and Hossner, 1985; Sommer et al. 2004). Sin embargo, la ventaja económica $(45-46 \% \mathrm{~N})$ asociada a su uso se ve reducida en varias situaciones comunes $\mathrm{y} / \mathrm{o}$ de creciente importancia en Chile, en las que la incorporación no es factible; entre otras, la segunda aplicación en cultivos anuales no escardados, la fertilización de mantención en praderas, su aplicación tanto en cero como en mínima labranza, las aplicaciones aéreas en silvicultura. En estas condiciones, la posibilidad de emplear urea queda supeditada al nivel de riesgo de volatilización existente, particularmente en el horizonte superficial de los suelos.

Las pérdidas de $\mathrm{NH}_{3}$ a partir de urea es entre un $18-30 \%$ del $\mathrm{N}$ aplicado con contenidos de agua suficientes en el suelo para la hidrólisis de la urea. Estos valores son comparables a los informados por Reynolds and Wold (1987) con pérdidas entre un 10-46\%, los que correlacionan con el porcentaje de arcilla, $\mathrm{N}$ total, capacidad de intercambio catiónico, $\mathrm{C}$ orgánico, ión $\mathrm{H}^{+}$, capacidad tampón y actividad de la ureasa del suelo.

Considerando que la pérdida real de $\mathrm{NH}_{3}$, a partir de urea, depende de factores del cultivo, de suelo y meteorológicos (Bolan et al., 2004; Sommer et al., 2004), su medición ha sido abordada por métodos diversos que en general presentan inconvenientes operativos, costos de ejecución altos e incertidumbre seria en la cuantificación de las tasas de pérdidas reales; esto último particularmente, debido a los errores que suelen asociarse entre la información obtenida en un laboratorio y su representación en condiciones de campo (Shirmohammadi et al., 2005).

No obstante, como alternativa a lo anterior, se puede plantear como suficiente, la determinación del potencial de volatilización de un suelo con el propósito de evaluar $a$ priori, el riesgo asociado en una aplicación superficial no incorporada de urea. En este sentido varios autores (Jury, 1999; Lin, 2003) destacan que sería muy beneficioso si los mecanismos y ciertas vías de flujo de ciertas sustancias (materiales fertilizantes, por 
ejemplo) en los suelos pueden ser determinados a partir de sus propiedades. Metodologías similares a las de este trabajo han sido utilizadas para caracterizar lixiviación (Kissel et al., 1982) y desnitrificación (Engle and Tiffany, 1984). En particular, para acidificación, Noble et al. (2002) emplean funciones de pedotransferencia (FPT) en la predicción de la capacidad de tamponamiento del $\mathrm{pH}$ en los suelos, en tanto que Minasny et al. (2005) las orientan a la obtención de mapas de almacenamiento de C orgánico. Las FPT, en un contexto no espacial, utilizan propiedades simples de suelo (a menudo encontradas en los estudios de suelo) determinadas con un nivel suficiente de certeza, permitiendo inferir información más compleja, desconocida o relativamente difícil de medir, con un mínimo de incertidumbre. Aunque estas funciones no pueden reemplazar a las mediciones directas de algunas propiedades edáficas, sí permiten mejorar la interpolación de información de terreno, es decir, extenderla desde un nivel de pedón hasta unidades cartográficas más amplias.

La investigación orientada a volatilización de amoníaco a partir urea en los suelos de Chile tiene una larga trayectoria. No obstante que en sus inicios los trabajos se efectuaron en condiciones controladas (Campillo, 1982; Aravena, 1984, por ejemplo), en los últimos años se han realizado avances notables en esta materia, aunque gran parte de las investigaciones (Núñez, 2008; Salazar et al., 2007; Vidal y Chamorro, 2005) se han concentrado en suelos del sur del país.

En consecuencia, el objetivo de este trabajo fue detectar y delinear zonas con elevado potencial de volatilización de $\mathrm{NH}_{3}$, a partir de urea granular aplicada superficialmente a suelos de la zona central de Chile, empleando información analítica estándar, proveniente de los estudios agrológicos, y funciones de pedotransferencia lógicamente vinculadas para estimar algunas variables determinantes del proceso.

\section{MATERIALES Y MÉTODOS}

\section{Cobertura del estudio}

El área estudiada en la zona central de Chile $\left(33^{\circ} 00^{\prime}-34^{\circ} 12^{\prime} \mathrm{LS}\right.$ y $\left.70^{\circ} 27^{\prime}-71^{\circ} 33^{\prime} \mathrm{LW}\right)$ corresponde principalmente a la Región Metropolitana con una superficie cercana a 400.000 ha. Los suelos presentes en esta área, según Series de suelo reconocidas (CIREN, 1996), son en su mayoría Inceptisoles y Entisoles (74\%) seguidas por Alfisoles, Vertisoles y Mollisoles (26\%). La Región presenta un clima templado cálido con lluvias invernales y 7 a 8 meses de estación seca. El área se selecciona considerando que cuenta con información cartográfica de suelos detallada (escala 1:20.000) a nivel de Fases y una analítica estándar de sus suelos (CIREN, 1996), suficientes para los propósitos planteados.

\section{Modelo conceptual}

Como se ha señalado, si bien las pérdidas reales de urea por volatilización dependen de numerosos factores, tales como manejo de los suelos y meteorológicos, un análisis de la dinámica que experimenta el N-urea en el suelo, en aplicaciones superficiales difusas, permite considerar que a) el potencial de volatilización depende fundamentalmente de variables de suelo y b) que éstas están asociadas al espacio físico conocido como micrositio (Hauck, 1984), con propiedades idénticas a las de la capa arable. La Figura 1 muestra esquemáticamente las interrelaciones entre los componentes y procesos involucrados, apreciándose que las variables de suelo determinantes de la volatilización son la actividad ureásica (AU), la capacidad de intercambio catiónico (CIC) y el pH final $\left(\mathrm{pH}_{f}\right)$ post-hidrólisis, definido por el $\mathrm{pH}$ inicial $\left(\mathrm{pH}_{i}\right)$ y la capacidad tampón contra alcalinización (CT). Estas variables fueron utilizadas como parámetros de clasificación del potencial de volatilización. 


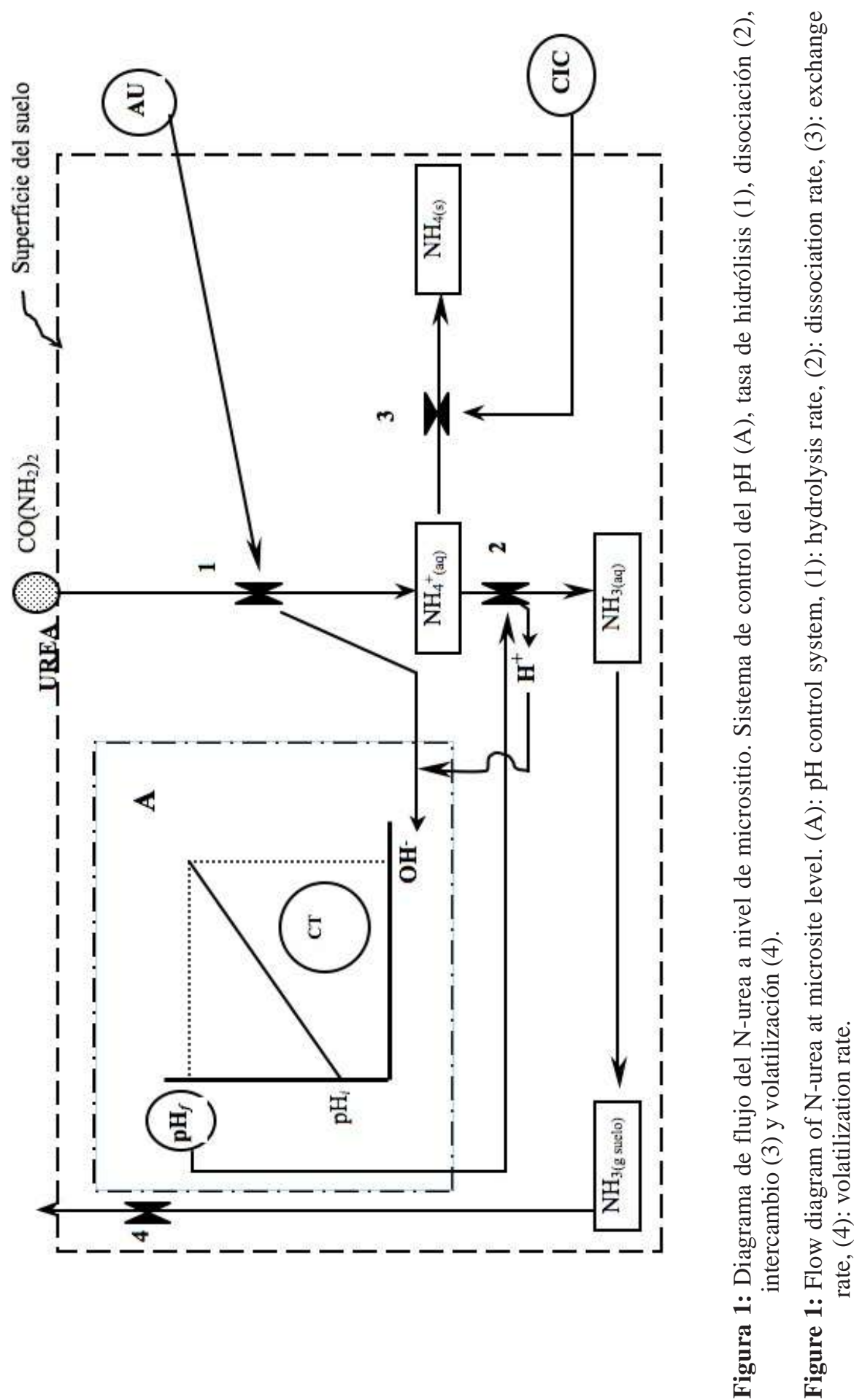




\section{Parámetros de clasificación}

Para confeccionar el registro completo de ellos en la zona en estudio, se utilizó la analítica de caracterización de los pedones correspondientes a las 82 Series de suelos que poseen analítica completa publicada. De la caracterización original se empleó directamente la CIC como parámetro de clasificación y los parámetros de clasificación $\mathrm{AU}$ y $\mathrm{pH}_{f}$, se determinaron mediante funciones de pedotransferencia (FPT) empleando regresión múltiple paso a paso (stepwise). Para la generación de las FPT requeridas, se utilizaron como variables independientes (predictoras) propiedades de una selección de 14 Series de suelo (importantes agrícolamente, de gran extensión y que cubren tanto la zona como el espectro de tipos de suelo de ella), en cuyas unidades cartográficas representativas se tomó una muestra compuesta de la capa arable $(0-20 \mathrm{~cm})$, en 1 ha de superficie. Salvo la capacidad tampón CT (Benavides et al., 1992) y los valores de actividad ureásica (AU) que corresponden a los informados por Casanova y Benavides (1995), se determinó a las 14 muestras de suelo sus propiedades predictoras, siguiendo las mismas metodología empleadas en el estudio agrológico del área (Black, 1965; Dewis y Freitas, 1970). Las propiedades fueron, los separados texturales (hidrómetro de Bouyoucos), el contenido de agua en equilibrio a 30 y $1.500 \mathrm{kPa}$ (olla y plato de presión), el contenido de materia orgánica (Walkley y Black), la capacidad de intercambio catiónico (acetato de sodio 1 $\mathrm{N}$ a pH 8,2), el pH en agua (relación 1:1), la conductividad eléctrica (extracto de saturación), el contenido de $\mathrm{CaCO}_{3}$ equivalente (neutralización ácida).

Funciones de pedotransferencia La obtención de las FPT para predecir CT y AU se hizo para un nivel de selección del $15 \%$, aplicando la transformación de Bliss y utilizando el programa estadístico Statgraphics (STSC, 1987).
La capacidad tampón (CT) medida corresponde a la ejercida contra la alcalinización del suelo inducida por la hidrólisis de urea, considerando que este proceso puede generar inicialmente un alza importante de $\mathrm{pH}$ a nivel de micrositio. La CT del suelo, representada por factores de tamponamiento, $\mathrm{BOH}_{1}$ y $\mathrm{BOH}_{2}$, se expresó como dpH $\left(\mathrm{cmol} \mathrm{OH}^{-1}\right)^{-1} \mathrm{~kg}^{-1}$ suelo, donde $\mathrm{dpH}$ corresponde a la variación del $\mathrm{pH}$ del suelo asociada a la alcalinidad originada por la hidrólisis de la urea contenida en un gránulo de tamaño estándar. Las FPT informadas son las siguientes:

$\mathrm{BOH}_{1}=45,11+1,29$ (a) - 3,12 (MO); $R^{2}: 0,87$

$\mathrm{BOH}_{2}=80,45-0,42(\mathrm{~A})+0,30(\mathrm{~L})$ - 6,89 $\left(\mathrm{pH}_{i}\right)-1,55(\mathrm{CE}) ; \mathrm{R}^{2}: 0,93$

las cuales se emplearon en el modelo:

$\mathrm{pH}_{f}=\mathrm{pH}_{i}+2,00\left(\mathrm{BOH}_{1}\right)+0,61\left(\mathrm{BOH}_{2}\right)$

La unidad de expresión de la AU del suelo fue $\mu \mathrm{g} \mathrm{N}$-urea $\mathrm{g}^{-1} \mathrm{~h}^{-1}$ (a $37^{\circ} \mathrm{C}$ ). La función de pedotransferencia obtenida fue:

$\mathrm{AU}=-1,47+6,77$ (M.O) + 1,84 (CIC); $R^{2}: 0,80$

Así, los valores de ureasa responden a su estabilización por la materia orgánica y los contenidos de arcilla (Chakrabarti et al., 2004, Corstanje et al. 2007).

\section{Umbrales del potencial de volatilización}

El potencial de volatilización de 82 Series de suelos del área estudiada, fue establecido en base a umbrales críticos definidos para los parámetros de clasificación: capacidad de intercambio catiónico (CIC), pH posthidrólisis estimado o $\mathrm{pH}$ final $\left(\mathrm{pH}_{f}\right)$ y niveles de actividad ureásica en la zona (AU). Dichos umbrales fueron establecidos a partir de la literatura $\left(\mathrm{CIC}\right.$ y $\mathrm{pH}_{f}$ ) y en función del rango de valores obtenidos para el área bajo 
estudio (AU). Posteriormente y bajo el supuesto que las propiedades de las unidades cartográficas representativas de cada Serie de suelo, son extrapolables al resto de las fases de una Serie, se procedió a agrupar según nivel de riesgo potencial a la totalidad de las unidades cartográficas del área comprometida.

\section{Esquema metodológico}

En la Figura 2 se sintetiza la metodología seguida para obtener zonas con distintos potenciales de volatilización, a partir de urea aplicada superficialmente a los suelos en la zona central de Chile.

\section{RESULTADOS Y DISCUSIÓN}

\section{Umbrales críticos}

Los criterios para definir umbrales críticos de los parámetros empleados en la clasificación de los suelos según su potencial de volatilización, variaron acorde a la naturaleza de éstos. Al respecto, Faurie et Bardin (1979) y O’Toole et al. (1985) concluyen que la volatilización es despreciable con una CIC superior a 20 $\mathrm{cmol}_{(+)} \mathrm{kg}^{-1}$, pero ésta podría aumentar de $20 \%$ a $75 \%$ del $\mathrm{N}$ aplicado, cuando la CIC del suelo desciende por debajo de 10 $\mathrm{cmol}_{(+)} \mathrm{kg}^{-1}$. En este sentido, Keller and Mengel (1986) precisan que los valores de volatilización casi se triplican en suelos franco arenoso con CIC de $7 \mathrm{cmol}_{(+)} \mathrm{kg}^{-1}$ respecto de suelos franco limosos con CIC de $12 \mathrm{cmol}_{(+)} \mathrm{kg}^{-1}$. No obstante, las altas tasas de volatilización informadas en suelos de textura gruesa no solo obedecen a una escasa retención del amonio por parte del suelo sino a la escasa capacidad tampón contra el $\mathrm{pH}$ que manifiestan estos suelos, particularmente durante la hidrólisis del fertilizante.

Con relación a la $\mathrm{AU}$, no existen actualmente criterios para definir cuantitativamente clases de actividad ureásica; esto es, no se ha hecho ningún intento de relacionar contenidos enzimáticos, medidos en las condiciones de los test usuales (concentración de sustrato, tiempo y temperatura de incubación), con las tasas de hidrólisis observables bajo condiciones reales. Luego, a fin de lograr una clasificación relativa de la capacidad hidrolítica de los suelos del área, se procedió a dividir el rango estimado de AU (Casanova y Benavides, 1995) en dos clases de actividad ureásica ( $\geq$ û $\mathrm{y}<$ û, donde û es el promedio medido en la zona estudiada), bajo el supuesto que los valores más altos están necesariamente asociados a niveles enzimáticos mayores $\mathrm{y}$, por lo tanto, a tasas hidrolíticas mayores.

A su vez, los valores de $\mathrm{pH}_{f}$ pueden clasificarse (Fenn and Hossner, 1985), en dos clases: sobre y bajo $\mathrm{pH}$ 7,5 en función de la estabilidad del ión $\mathrm{NH}_{4}{ }^{+}$en solución. Sin embargo, si se tiene presente la depresión del valor del $\mathrm{pH}$ por el efecto salino (Bohn, 1979) post hidrólisis generado por el fertilizante, a nivel de micrositio, debería considerarse 0,5 unidades adicionales, es decir, pH 8. Este hecho es confirmado por Sommer et al. (2004) quien señala que bajo este umbral, el $95 \%$ del $\mathrm{N}$ estaría en la forma de $\mathrm{NH}_{4}^{+}$pudiendo ser intercambiado con otros cationes, en tanto que sobre este valor (a nivel de micrositio) una gran proporción estaría como $\mathrm{NH}_{3}$.

En consecuencia, la clase con mayor potencial de pérdida amoniacal, asociada a aplicaciones superficiales difusas de urea en el área estudiada, incluye suelos que tienen como característica común: una actividad ureásica $\geq 40$ ug N-urea $\mathrm{g}^{-1} \mathrm{~h}^{-1}$ a $37^{\circ} \mathrm{C}$; una $\mathrm{CIC} \geq 20 \mathrm{cmol}_{(+)} \mathrm{kg}^{-1}$ y un valor de $\mathrm{pH}_{f} \geq 8,0$.

\section{Potencial de volatilización de los suelos en la zona estudiada}

Dadas las complejas interacciones entre variables de suelo, de manejo y ambientales que afectan las pérdidas reales de amoníaco, desde urea granular aplicada a la superficie de los suelos, es posible suponer que para la zona estudiada, se deberían mantener las diferencias impuestas por las variable de suelo, alcanzando éstas la connotación de pérdidas potenciales. Al respecto, Sommer et al. (2004) señalan que las pérdidas totales 


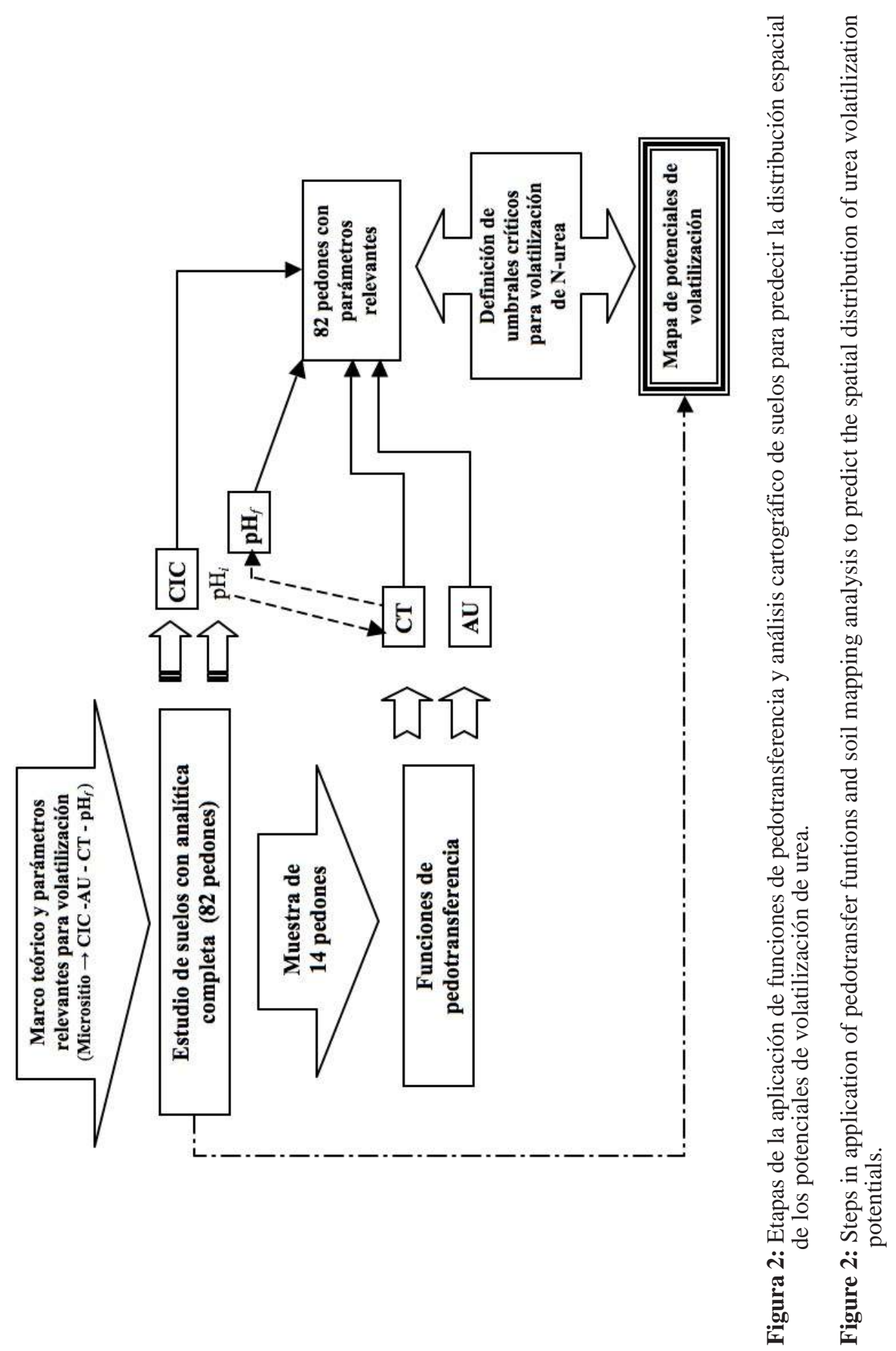


Cuadro 1: Series de suelos definidas como de más alto riesgo de volatilización.

Table 1: Soil Series with the highest risk of ammonia volatilization.

\begin{tabular}{|l|c|c|c|c|c|c|r|}
\hline Serie de suelos & $\mathbf{A U}$ & $\mathbf{B O H}_{\mathbf{1}}$ & $\mathbf{B O H}_{\mathbf{2}}$ & $\mathbf{C I C}$ & $\mathbf{p H}_{\boldsymbol{i}}$ & $\mathbf{p H}_{\boldsymbol{f}}$ & Superficie \\
\hline & $\mu \mathrm{g} \mathrm{N}-\mathrm{urea}^{-1} \mathrm{~h}^{-1}$ & $\mathrm{dpH}\left(\mathrm{cmol} \mathrm{OH}^{-1} \mathrm{~kg}^{-1}\right.$ & $\mathrm{cmol}_{(+)} \mathrm{kg}^{-1}$ & & & \multicolumn{1}{c|}{$(\mathrm{ha})$} \\
\hline Barrancas & 51,8 & 57,6 & 25,4 & 16,8 & 7,8 & 9,1 & 923,0 \\
\hline Clarillo & 45,8 & 58,6 & 29,9 & 17,0 & 7,6 & 9,0 & $1.222,0$ \\
\hline Colina & 41,0 & 73,5 & 31,6 & 19,7 & 7,4 & 9,1 & $2.573,7$ \\
\hline Cuesta Vieja & 46,4 & 72,3 & 38,9 & 18,3 & 6,5 & 8,2 & $1.334,7$ \\
\hline Cholqui & 53,6 & 46,1 & 26,7 & 19,0 & 7,7 & 8,8 & $2.542,8$ \\
\hline Huingán & 41,8 & 64,0 & 24,7 & 15,8 & 7,9 & 9,3 & 711,8 \\
\hline Los Morros & 44,5 & 56,1 & 25,5 & 15,5 & 8,1 & 9,4 & $1.685,7$ \\
\hline Maipo & 40,0 & 61,6 & 22,0 & 16,3 & 8,0 & 9,4 & $33.212,7$ \\
\hline Pintué & 40,5 & 81,7 & 35,4 & 13,6 & 6,7 & 8,5 & $6.977,8$ \\
\hline San Diego & 49,5 & 55,9 & 26,8 & 17,7 & 7,7 & 9,0 & $6.421,3$ \\
\hline Santiago & 43,1 & 65,7 & 28,9 & 14,4 & 7,8 & 9,3 & $17.758,3$ \\
\hline Sta. Rita de Casablanca & 44,8 & 81,9 & 35,6 & 11,9 & 6,3 & 8,2 & $1.193,8$ \\
\hline Tapihue & 42,8 & 78,5 & 32,6 & 15,2 & 6,9 & 8,7 & $6.946,5$ \\
\hline Valdivia de Paine & 49,1 & 58,1 & 30,0 & 15,9 & 8,0 & 9,4 & $4.534,8$ \\
\hline Total & & & & & & $\mathbf{8 8 . 0 3 8 , 9}$
\end{tabular}

AU= actividad ureásica

$\mathrm{BOH} 1$ y $\mathrm{BOH} 2=$ coeficiente de tamponamiento

$\mathrm{CIC}=$ capacidad de intercamnio catiónico

pHi y $\mathrm{pHf}=\mathrm{pH}$ inicial y final (post hidrólisi), respesctivamente

pueden no ser afectadas por cambios en la temperatura; consecuentemente, la pérdida total puede relacionarse al potencial de pérdidas dados por el suelo y otras variables.

Así, la aplicación de los umbrales permite establecer que en la zona estudiada, el área con más alto riesgo de pérdida amoniacal por volatilización asociada a aplicaciones superficiales difusas de urea, alcanza un valor de 88.039 ha, equivalente al $22,4 \%$ del área cartografiada en la zona y corresponde casi en su totalidad a suelos del Orden Mollisol (Cuadro1). Este hecho responde a las características de estos suelos en la zona central de Chile: poseen un epipedón mólico que ocupa más de un tercio del espesor combinado de los horizontes A y $\mathrm{B}$ o tiene más de $25 \mathrm{~cm}$ de espesor; la estructura no es dura o muy dura en seco; son ricos en bases y el calcio es el catión extractable principal en dichos horizontes; dominan los minerales de arcilla cristalinos de CIC moderada a alta; poseen menos de $30 \%$ de arcilla en los $50 \mathrm{~cm}$ superficiales y no poseen grietas (Luzio et al., 2006).

La distribución del área comprometida se muestra en la Figura 3 y se ubica espacialmente en las comunas más próximas, al oeste y sur-oeste, de la ciudad de Santiago. Por otra parte, si se incluyen 20 Series de suelo adicionales, que no satisfacen el requerimiento definido tentativamente para la clase de potencial de volatilización alto, y que ocupan una superficie de 120.869 ha (30\% del total cartografiado), el área total se extiende a 208.908 ha y están constituidas por suelos con riesgo de pérdidas potenciales alto a probablemente alto. Esta última superficie no se incluye en la Figura 3, pero presenta una distribución más amplia. 


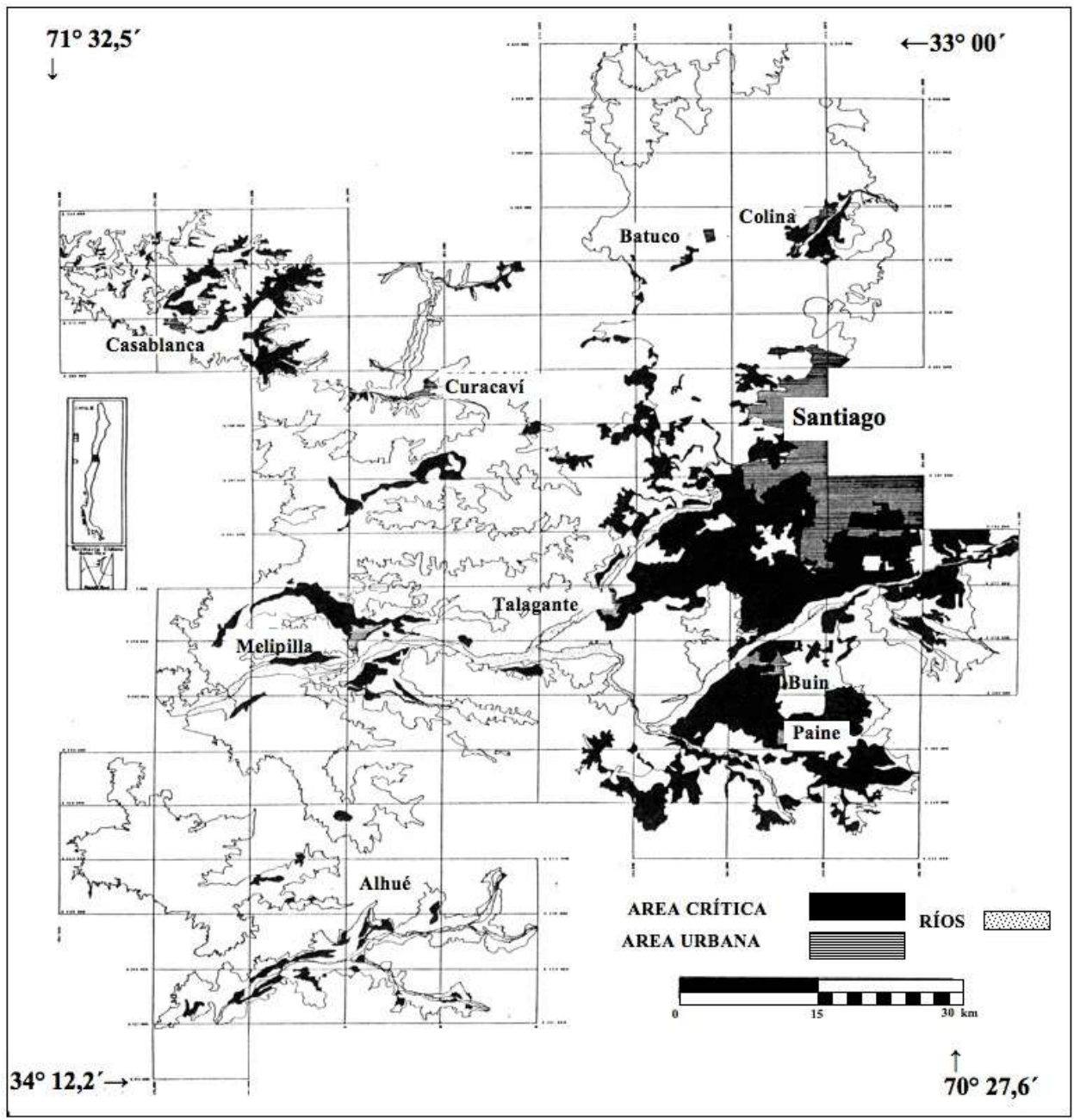

Figura 3. Suelos con alto potencial de volatilización de urea en la zona central de Chile.

Figure 3. Soils with high urea volatilization potential in the central zone of Chile. 


\section{CONCLUSIONES}

Mediante la obtención de FPT para los parámetros de clasificación, capacidad tampón y actividad ureásica, más información agrológica estándar (variables de predicción), es posible cartografiar en un área determinada la zona con alto riesgo potencial de emisión de $\mathrm{NH}_{3}$ a la atmósfera, asociada al uso de urea granular en aplicaciones superficiales difusas.

Sin considerar factores importantes como los meteorológicos (particularmente lluvias, que afectan la pérdida real) y de manejo de los suelos, la aplicación del método descrito permite identificar áreas de alto riesgo en una extensa zona agrícola de Chile central, con una adecuada capacidad discriminatoria, dando como resultado que la superficie con un alto potencial de volatilización alcanza a 88.039 ha y compromete principalmente a suelos del Orden Mollisol, cuyas propiedades intrínsecas propenden dicha pérdida.

La metodología propuesta, en base a umbrales críticos, representa una aproximación apropiada, replicable y de bajo costo para la selección espacial de fuentes nitrogenadas y métodos de aplicación, particularmente si se busca minimizar pérdidas económicas e impactos ambientales, relacionados al uso de la urea como fertilizante.

\section{REFERENCIAS}

Aravena, P.C. 1984. Transformaciones de la urea en tres series representativas de la provincia de Quillota. Tesis de Ingeniero Agrónomo. Quillota, Universidad Católica de Valparaíso. Facultad de Agronomía. 66 p.

Benavides, C., Casanova, M., Rustom, A. 1992. Capacidad de tamponamiento contra alcalinidad en suelos de las regiones $\mathrm{V}$ y Metropolitana de Chile. Agricultura Técnica 52, 426-430.
Black, C.A. 1965. Methods of soil analysis. Part 2. Chemical and microbiological properties. American Society of Agronomy. Agronomy 9. 1.572 p.

Bolan, N., Saggar, S., Luo, J., Bhandral, R., Singh, J. 2004. Gaseous emissions of nitrogen from grazed pastures: processes, measurements and modeling, environmental implications, and mitigation. Advances in Agronomy 84, 37-102.

Bohn, H.L., Bian, L., McNeal, S., O’Connor. 1979. Soil Chemistry. Wiley, New York. 329 p.

Campillo, R. 1982. Efecto acidificante de las transformaciones de la urea en dos Andisoles de la Región de Los Lagos. Tesis de Magíster, Universidad Católica de Chile. Facultad de Agronomía. $137 \mathrm{p}$.

Casanova, M., Benavides, C. 1995. Actividad de la ureasa en suelos de la zona central de Chile. Agricultura Técnica $55,154-158$.

Chakrabarti, K., Sinha, N., Chakraborty, A., Bhattacharyya, P. 2004. Influence of soil properties on urease activity under different agro-ecosystems. Archives of Agronomy and Soil Science 50, 477-483.

CIREN. 1996. Estudio agrológico Región Metropolitana. Centro de Información de Recursos Naturales. Descripciones de suelos, Materiales y símbolos. Publicación $\mathrm{N}^{\circ} 115.425$ p.

Corstanje, R., Schulin, R., Lark, R. 2007. Scale-dependent relationships between soil organic carbon and urease activity. European Journal of Soil Science 58, 1087-1095. 
Dewis, J., F. Freitas. 1970. Método físicos y químicos de suelos y aguas. Food and Agricultura Organization, Boletín de Suelo $\mathrm{N}^{\circ} 10.252$ p.

Drinkwater, L., Snapp, S. 2007. Nutrients in Agroecosystems: rethinking the management paradigm. Advances in Agronomy 92, 163-186.

Engle, C., Tiffany, C. 1984. Rating eastern Washington soil for potential $\mathrm{N}$ losses. Washington State University, Extention Bulletin 1258. 9 p.

Faurie, G., Bardin, R. 1979. La volatilization de l'amoniac. I. Influence de la nature du sol et des composés azotés. Annales Agronomiques 30, 363-385.

Fenn, M., Baron, J., Allen E., Rueth, H., Nydick, K., Geiser, L. 2003. Ecological effects of nitrogen deposition in the Western United States. Bioscience 53, 404-420.

Fenn, L., Hossner, L. 1985. Ammonia volatilization from ammonium or ammonium-forming nitrogen fertilizer. Advances in Soil Science 1, 124-169.

Galloway J., Aber, J., Erisman, J., Seitzinger, S., Howarth, R., Cowling, E. 2003. The nitrogen cascade. Bioscience 53, 341356.

Howard D., Newman, M., Essington, M., Percell, W. 2002. Nitrogen fertilization of conservation tilled wheat. I. Sources and application rates. Journal of Plant Nutrition 25, 1315-1328

Ji-Qin Ni , Heber, A.J. 2008. Sampling and Measurement of Ammonia at Animal Facilities. Advances in Agronomy 98, 201-269.
Jury, W.A. 1999. Present directions and future research in vadose zone hydrology, In: M.B. Parlange, J.W. Hopmans.W. (eds.): Vadose zone hydrology. Cutting across disciplines. Oxford University Press, New York. p. 433-441.

Keller, G.D., Mengel, D.B. 1986. Ammonia volatilization from nitrogen fertilizers surface applied to no-till corn. Soil Science Society of American Journal 50, 1060-1063.

Keller, D.K., Brummer, E.C. 2002. Putting food production in context: toward a postmechanistic agricultural ethic. BioScience 52, 264-271.

Kissel, D., Bidwell, O., Kienz, J. 1982. Leaching classes of Kansas soils. Kansas State University, Bulletin 641. 12 p.

Lin, H. 2003. Hydropedology: bridging disciplines, scales, and data. Vadose Zone Journal, 2:1-11.

Luzio, W., Casanova, M., Vera, W. 2006. Clasificación de suelos, In: Luzio W., Casanova M. (Eds.): Avances en el conocimiento de los suelos de Chile. Universidad de Chile y Servicio Agrícola y Ganadero (SAG). Santiago. p. 241286.

Malhi, S., Grant, C., Johnston, A., Gill, K. 2001. Nitrogen fertilization management for no-till cereal production in the Canadian Great Plains: a review. Soil Tillage Research 60, 101-122.

Minasny, B., McBratney, A., MendonçaSantos, M., Odeh I., Guyon, B. 2005. Prediction and digital mapping of soil carbon storage in the Lower Namoi Valley. Australian Journal of Soil Research 44(3), 233-244. 
Noble, A., Middleton, C., Nelson, P., Rogers, L. 2002. Risk mapping of soil acidification under Stylosanthes in northern Australian rangelands. Australian Journal of Soil Research 40(2), 257-267.

Nuñez, P. 2008. Efecto de la frecuencia e intensidad de pastoreo en las pérdidas de nitrógeno en una pradera permanente del sur de Chile. Tesis de Doctor en Ciencias de Recursos Naturales, Universidad de La Frontera, Temuco, Chile. 186 p.

O’Toole, P., McGarry, S., Morgan, M. 1985. Ammonia volatilization from urea-treated pasture and tillage soils: effects of soil properties. Journal of Soil Science 36, 613-620.

Reynolds, C. M., Wold, D. C. 1987. Influence of urease activity and soil properties on ammonia volatilization from urea. Soil Science 143, 418-425.

Salazar, F., Alfaro, M., Lagos, J., Williams, J., Ramírez, L., Valencia, E. 2007. Volatilización de amoníaco por la aplicación de urea en una pradera permanente en Osorno, In: González H., Iraira H. (Eds.): Proceeding XXXII Congreso Anual de la Sociedad Chilena de Producción Animal. 14 al 16 noviembre. Frutillar, Chile. p. 53-54.
Schlesinger, W.H., Hartley, A. E.. 1992. A global budget for atmospheric $\mathrm{NH}_{3}$. Biogeochemistry 15, 191-211.

Shirmohammadi, A., Djodjic, E., Bergström, L. 2005. Scaling issues in sustainable management of nutrient losses. Soil Use and Management 21, 160-166.

Sommer, S., Schjoerring, J., Denmead, O. 2004. Ammonia emission from mineral fertilizers and fertilized crops. Advances in Agronomy 82, 558-622.

STSC. 1987. User's Guide Statgraphics. Graphic Software System. Statistical Graphics Systems Corporation Inc.Rocville, MD.

Vidal, I., Chamorro, S. 2005. Pérdida de nitrógeno por volatilización a partir de la aplicación superficial de urea, In: Casanova, M. (Ed.): Proceeding X Congreso Nacional de la Ciencia del Suelo. Facultad de Ciencias Agronómicas, Universidad de Chile. Santiago, 16-18 de noviembre. Boletín de la Sociedad Chilena de la Ciencia del Suelo $N^{\circ} 21$. p. 138-138. 